\title{
Diagnosis and Management of Bilateral Uterine Arteries Aneurysm during Puerperium
}

\author{
Dr. Amir Elnahas ${ }^{1}$, Mohammed Khidir Tayfoor ${ }^{2}$ and Salma Mohammed Hassan Eltayeb ${ }^{3 *}$ \\ ${ }^{1}$ Consultant Obstetrician and Gynecologist at Sidra medical and research center, MD in obstetrics\&gynecology, FRCOG1 \\ ${ }^{2}$ Consultant interventional vascular radiologist and consultant interventional neurologist, MD, Radiology (U of K)-fellowship IR, Jordan \\ ${ }^{3}$ Specialist of obstetrics and gynecology, Jordan
}

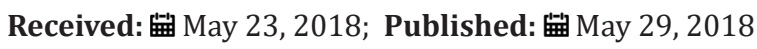

*Corresponding author: Dr. Salma Mohammed Hassan Eltayeb, Specialist of obstetrics and gynecology, Jordan, Tel: 00249911137869, Email: loma.2009@hotmail.com

\begin{abstract}
Background: Uterine artery pseudo aneurysm is a rare but it can be one of complication of cesarean section. If treated inadequately, it may cause profuse life-threatening postpartum hemorrhage. We report a rare case of bilateral uterine arteries pseudoaneurysm that lead to life threatening secondary postpartum hemorrhage.
\end{abstract}

Case: 25 years old, PI, she underwent category 1 caesarean delivery, 55 days later she experienced recurrent massive attack of secondary postpartum hemorrhage. The diagnosis of bilateral uterine arteries aneurysm was made by CT angiograph. It was successfully treated by selective embolization of the bilateral uterine arteries.

Conclusion: Rupture of aneurysm of the uterine artery can be a life-threatening condition. Clinical suspicious is the key point for prompt diagnosis. Diagnosis can achieve by angiography and embolization therapy can effectively stop the bleeding.

Keywords: Postpartum Hemorrhage; Pseudoaneurysm; Angiography; Embolization

Abbreviations: PPH: Postpartum Hemorrhage; UAP: Uterine Artery Pseudoaneurysms; D\&C: Dilatation and Curettage;

\section{Introduction \& Literature Review}

Postpartum hemorrhage remains one of the major causes of maternal morbidity and mortality. Secondary postpartum hemorrhage (PPH) is defined as excessive bleeding starting any time from 24 hours after delivery up to 12 weeks postpartum and most commonly occurring between 8 and 14 days postpartum. Common causes include retained products of conception, sub involution of the placental bed, and endometritis. Rare causes include pseudo aneurysm of uterine artery, arteriovenous malformations, and choriocarcinoma. Secondary PPH is rare. Until now secondary PPH has received very little attention probably because of its low incidence and because it causes more morbidity than mortality. However severe secondary PPH may sometimes become fatal [1]. A true aneurysm has all three layers of arterial wall, whereas pseudo aneurysm does not have all the three layers of arterial wall. Uterine artery pseudoaneurysms (UAP) are rare and potentially lifethreatening lesions. Their actual prevalence rate remains unknown.
The UAPs may occur after using an intrauterine device, traumatic delivery, pregnancy termination, cesarean section, manual placenta removal, forceps delivery, vacuum extraction, or dilatation and curettage (D\&C) [2]. The diagnosis of a uterine artery aneurysm can be made with ultrasound but when small, and when rupture has occurred, visualization may be difficult, CT scanning may be useful in identifying underlying arterial abnormality and it is often best demonstrated by angiography[3]. Arterial embolization should be considered as the treatment of choice for stable patients [4]. We report a case of left uterine artery aneurysm after an uncomplicated caesarean delivery in a patient with no surgical history.

\section{Case Report}

25 years old lady, Para 1 was transferred to our hospital 55 days post her delivery, with symptoms of recurrent excessive vaginal bleeding, she underwent category 1 caesarean delivery 
for fetal distress ,she was apparently asymptomatic for 35 days post operation, later she developed recurrent excessive vaginal bleeding , and was admitted in hospital and diagnosed as case of endometritis and received intravenous antibiotics, she developed the same condition several times in which she was treated with same policy. In her last admission, D\&C was done for the patient, but bleeding was not stop so laparotomy was done, compression of the uterus was done for 10 minute (bleeding was stopped), she received 14 unit of blood, and discharged in good condition. She was developed the same condition three times after the laparotomy, the last episode 6 days prior to her referring. Regarding her PMH, DH, FH were no significant.

When she came to the outpatient clinic she was well, moderately pale, pulse 72 , blood pressure 110/60.

Abdomen examination: was normal.

Speculum examination: no abnormality.

I. Her investigation revealed that: $\mathrm{Hb} 10 \mathrm{~g} / \mathrm{dl}$ TWBCs 14.1 Platelets 266

\section{RFT \&LFT were normal.}

III. Coagulation profile: $\mathrm{PT}=14.5$ seconds $\mathrm{PTT}=27$ seconds INR $=1.1$

IV. Serum Beta HCG: 0.5 IU/L.

V. U/S abdomen \&pelvis: normal anteverted uterus, endometrial thickness $=5 \mathrm{~mm}$, there was hypoechoic area, pulsatile at the site of caesarean section scar $=10 \mathrm{~mm}$ ? A/v shunt.

VI. CT angiograph: the left uterine artery is slightly enlarged compared to the right one and ended with abnormal tangle of vessels in the myometrium of the lower uterine segment on the anteriolateral aspect.

There is small lake of contrast on the medial aspect of the network of abnormal vessels; consistent with left uterine artery aneurysm, the right uterine artery is normal, rest of vasculature of the pelvis and abdomen are normal .please see the images below (Figure 1):

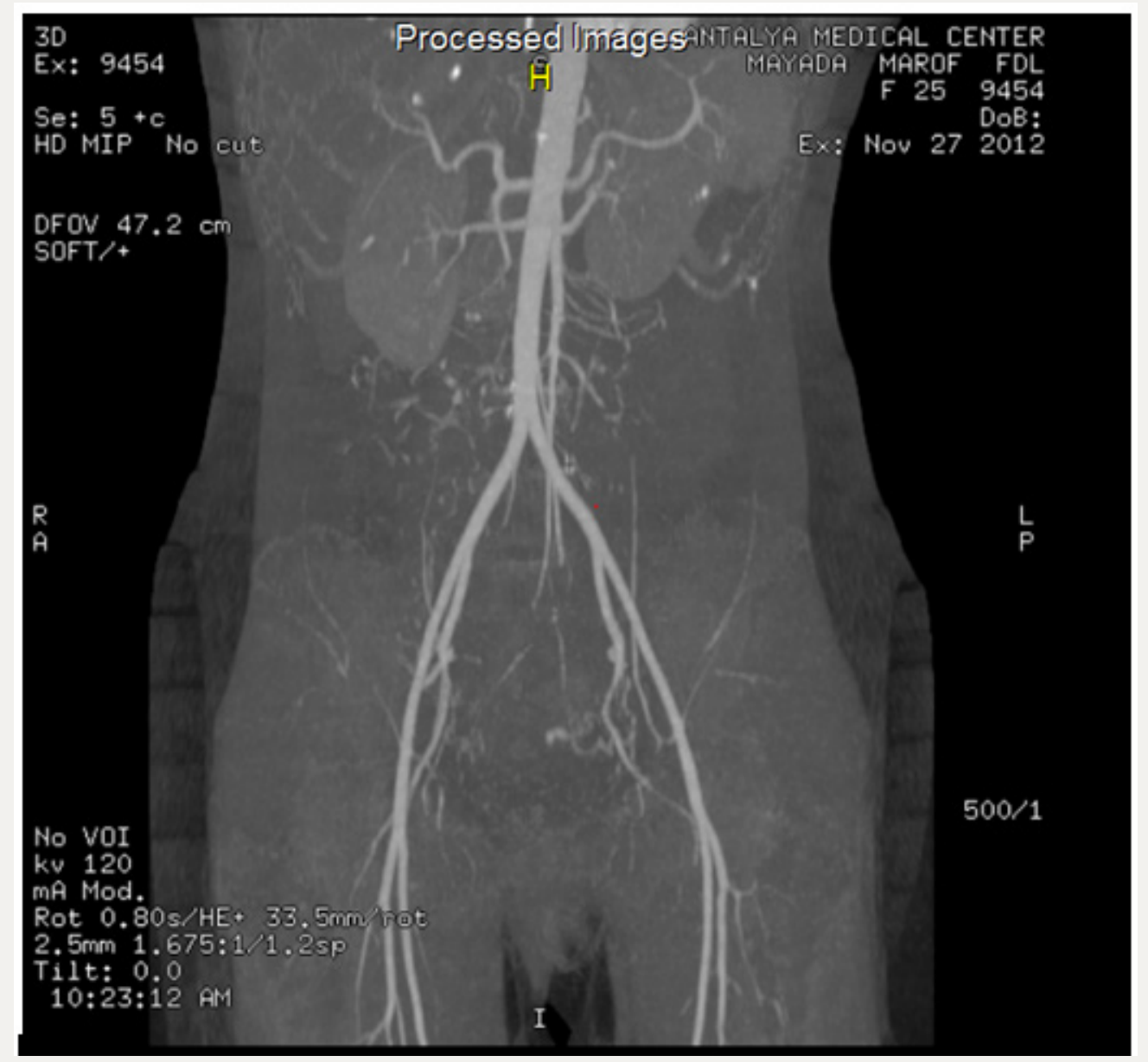

Figure 1. 
We sent our patient to the radiologist and he found bilateral uterine aneurysm. The woman's post procedural course was un eventful. She discharged two days after embolization.
Following images show the procedure: Angiography for both uterine arteries (Figure 2-5):

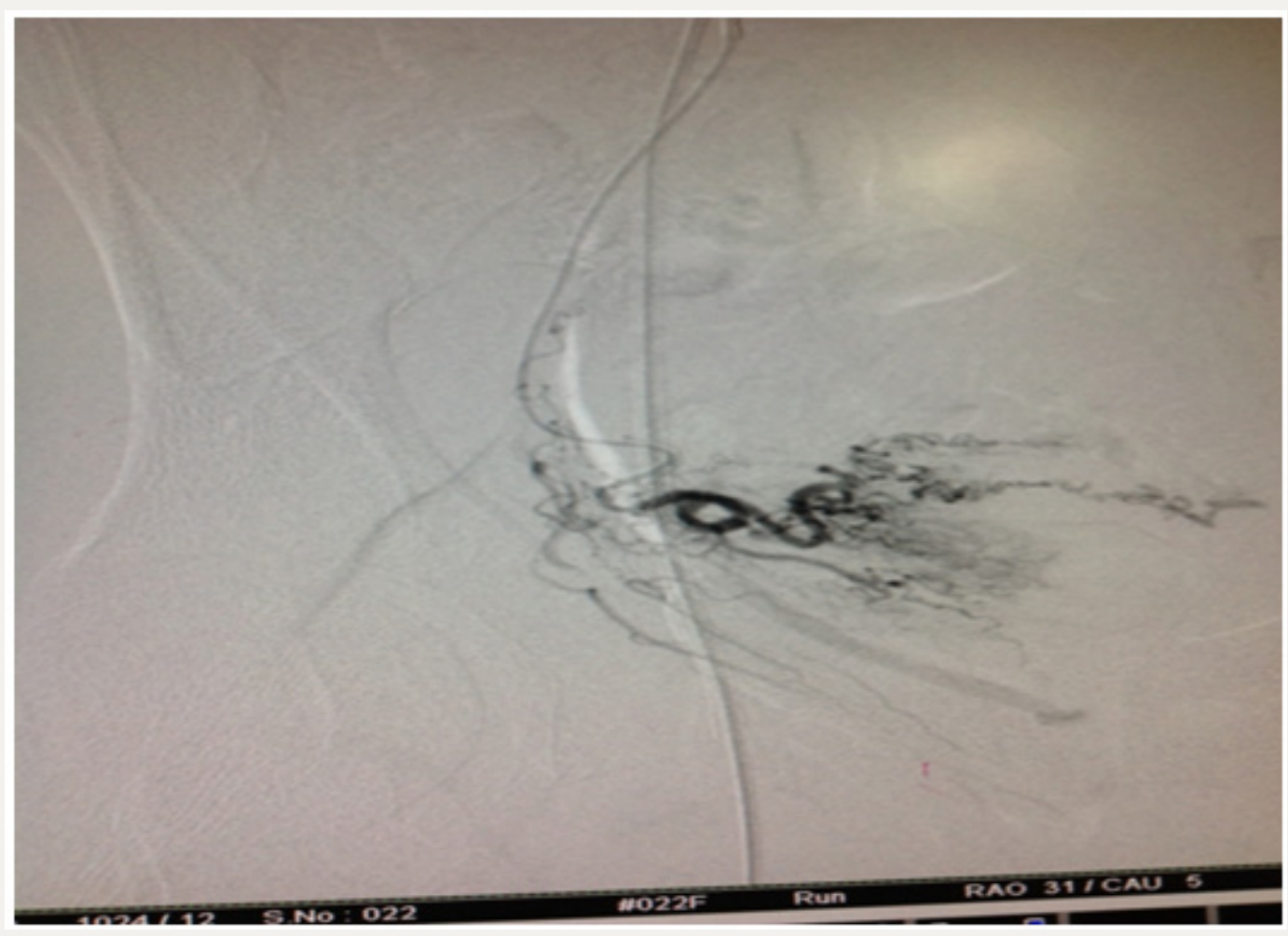

Figure 2: Right uterine artery (pre procedure).



Figure 3: Right uterine artery (post procedure). 


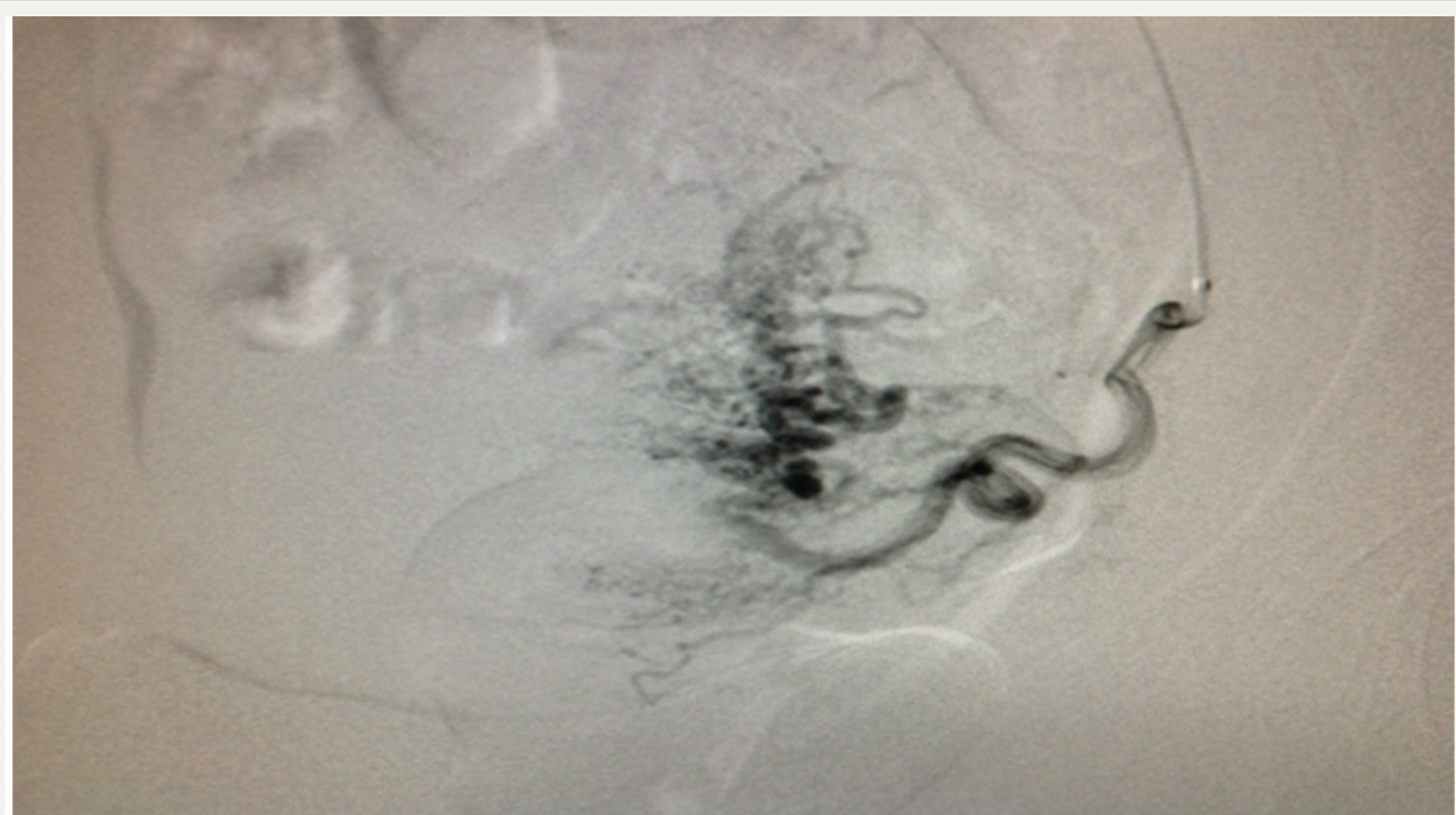

Figure 4: Left uterine artery (pre procedure).



Figure 5: Left uterine artery (post procedure). 


\section{Discussion}

Uterine vascular lesions are rare but potentially life-threatening. True uterine artery aneurysms are usually congenital and can be categorized as either fusiform or saccular. Fusiform aneurysms are characterized by localized dilatation of the vessel lumen to approximately twice the size of the proximal and distal portions. Saccular aneurysms are localized extrusions from the vessel that contain all three layers of vessel wall; a wide spectrum of vascular abnormalities can affect the uterine vasculature. These abnormal vascular diseases that affected the arteries were arteriovenous malformations, true aneurysms, and pseudo aneurysms [5]. Pseudo aneurysms and arteriovenous malformations are usually acquired. They are usually the consequence of uterine curettage or surgical trauma. Arterial pseudo aneurysms are complications of vascular injury. When a punctured or lacerated artery does not seal completely, blood escapes, dissects the adjacent tissues, and collects in perivascular areas. If this collection maintains communication with the parent vessel, a pseudo aneurysm can result. In obstetrics; trauma to the uterine arteries from caesarean section is the commonest cause of pseudo-aneurysm. This can be due to a lateral extension of the lower uterine segment incision and failure to secure the apex of the incision during repair, or if the operation was performed at advanced dilatation. However, pseudo aneurysms have also been reported after normal vaginal delivery, possibly due to a true congenital aneurysm. Pseudo aneurysm on gray- scale ultrasound appears as an anechoic sac which on Doppler appears as a blood-filled cystic structure with turbulent arterial flow .Angiography remains the gold standard imaging technique in diagnosing vascular malformations. Pseudo aneurysms usually show a blood-filled cystic structure with swirling arterial flow color under duplex Doppler ultrasound [6].

Acquired arteriovenous malformations are the result of trauma, malignancy, or infection that has resulted in a connection between an artery and an adjacent vein. In cases of arteriovenous malformations, color Doppler ultrasound shows an intense vascular tangle, whereas duplex Doppler ultrasound shows low-resistance, high-velocity arterial flow. Recognition of these abnormalities as the cause of hemorrhage is important, since these abnormalities can be treated safely and effectively with transcatheter arterial embolization but may be worsened by uterine curettage, precipitating massive uterine bleeding [5]. Although embolization is becoming the treatment modality of choice for preventing and controlling postpartum hemorrhage, its use depends on the availability of interventional radiologists and angiographic equipment, with rapid intervention significant morbidity and mortality associated with postpartum hemorrhage can become the thing of the past. With newer modalities of treatment available, hysterectomy for postpartum hemorrhage should a last resort [7].

\section{References}

1. Khong TY, Khong TK (1993) Delayed postpartum hemorrhage: A morphologic study of causes and their relation to other pregnancy disorders. Obstet Gynecol 82(1): 17-22.

2. Nagayama C, Gibo M, Nitta H, Uezato T (2011) Rupture of pseudoaneurysm after vaginal delivery successfully treated by selective arterial embolization. Arch Gynecol Obstet 283(1): 37-40.

3. Pelage JP, Soyer P, Repiquet D, Herbreteau D, Le Dref O, et al. (1999) Secondary postpartum hemorrhage: treatment with selective arterial embolization. Radiology 212(2): 385-389.

4. T Kuwata, S Matsubara, Y Kaneko, A Izumi, M Nakata, et al. (2010 ) Asymptomatic uterine artery pseudo aneurysm after cesarean section. Journal of Obstetrics and Gynecology Research 36(2): 405-410.

5. Greenfield LF, Mulholland MD, Oldham KT et al. eds. (1993) Vascular malformation and arteriovenous fistula. Surgery: Scientific Principles and Practice. Philadelphia: JB Lippincott Co pp 1741-1755.

6. Zimon AE, Hwang JK, Principe DL, Bahado-Singh RO. Pseudo aneurysm of the uterine artery. Obstetrics \& Gynecology 94(5): 827-830.

7. Spies JS, Pelage JP (2005) Uterine artery embolization and gynecologic embolotherapy. Philadelphia: Lippincott Williams \& Wilkins. pp. 3-18.

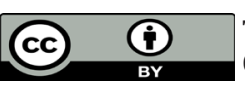

This work is licensed under Creative Commons Attribution 4.0 License

To Submit Your Article Click Here:

Submit Article

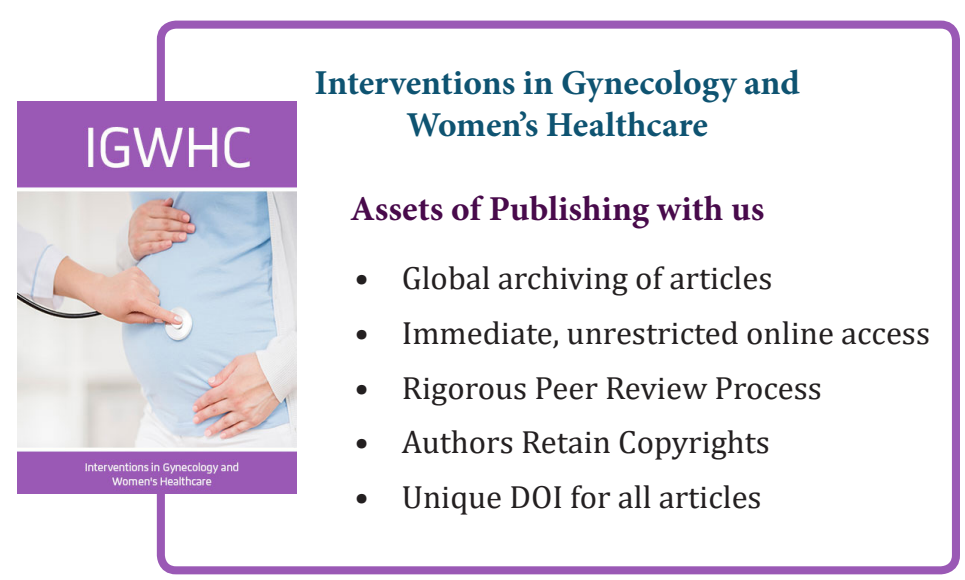

DOI: $10.32474 /$ IGWHC.2018.02.000131 